\title{
COMPORTAMENTO DAS GERAÇÕES Y E Z QUANTO A IMPORTÂNCIA DA EDUCAÇÃO FINANCEIRA
}

\author{
BEHAVIOR OF GENERATIONS Y AND Z REGARDING THE IMPORTANCE OF FINANCIAL \\ EDUCATION
}

\section{COMPORTAMIENTO DE LAS GENERACIONES Y E Z EN CUANTO A LA IMPORTANCIA DE LA EDUCACIÓN FINANCIERA}

Cláudio Luiz Chiusoli ${ }^{1}$, Thiago Ferreira Spiri², Ana Lucia Mendes ${ }^{3}$, Bruna Volski dos Santos ${ }^{4}$, Laryssa Latczuk Eurich $^{5}$

e311004

https://doi.org/10.47820/recima21.v3i1.1004

\section{RESUMO}

$O$ estudo é referente ao tema educação financeira e sua abordagem é sobre as gerações $Y$ e $Z$, considerando-se que há uma preocupação das diferentes gerações quanto ao futuro no campo profissional, financeiro e pessoal em relação à consciência da educação financeira. O objetivo é analisar a opinião e atitude das duas gerações, $\mathrm{Y}$ e Z, em relação à importância e ao conhecimento sobre a educação financeira. Foi realizada uma abordagem teórica caracterizando as duas gerações sobre educação financeira e finanças pessoais. Como método de pesquisa, foram realizadas coletas em cidades do interior do Paraná por meio da aplicação de 102 questionários, enviados via Google Forms, caracterizando amostragem não probabilística. Os principais achados apontam que $66,7 \%$ citam que têm conhecimento sobre administração financeira e $63,7 \%$ têm controle das finanças pessoais, e $89,2 \%$ alegam ter conhecimento da importância do planejamento para estabilidade financeira. $72,5 \%$ concordam que é possível fazer poupança com o salário atual, $41,2 \%$ concordam que conseguem ter acesso às informações sobre investimentos nas redes sociais e $75,5 \%$ assinalam que tem clareza de quanto economizar mensalmente para alcançar o objetivo proposto. Por fim, $78,4 \%$ concordam que têm conhecimento entre o que são gastos essenciais, necessários e supérfluos, $70,6 \%$ citam que têm conhecimento de quais gastos são mais importantes e 77,5\% concordam que têm condições de contribuir com as despesas domiciliares em casa. Baseado nos resultados obtidos, foi possível verificar que as duas gerações declaram ter um certo conhecimento sobre administração financeira, da mesma forma que demonstram a importância de poupar para o crescimento profissional e pessoal.

\section{PALAVRAS-CHAVE: Geração Z. Geração Y. Educação financeira}

\section{ABSTRACT}

The study is related to the topic of financial education and its approach is with the $Y$ and $Z$ generations and, it is evident that there is a concern of different generations about the future in the professional, financial and personal field in relation to the awareness of financial education. The objective is to analyze the opinion and attitude of the two generations, $Y$ and $Z$, in relation to the importance and knowledge of financial education. A theoretical approach characterizing the two generations, on financial education and personal finance, was carried out. As a research method, collections were carried out in cities in the interior of Paraná through the application of 102 questionnaires, sent via Google Forms, featuring a non-probabilistic sampling. The main findings indicate that $66.7 \%$ mention that they have knowledge about financial management and $63.7 \%$ have control of personal finances, and $89.2 \%$ claim to be aware of the importance of planning for financial stability. $72.5 \%$ agree that it is possible to save with the current salary, $41.2 \%$ agree that they can access information about investments on social networks and $75.5 \%$ indicate that they are clear about how much to save monthly to reach the proposed objective. Finally, $78.4 \%$ agree that they are aware of what are essential, necessary and superfluous expenses, $70.6 \%$ cite that they are aware of which expenses are most important and $77.5 \%$ agree that they are able to contribute to the expenses household

\footnotetext{
1 Pós-Doutor em Gestão Urbana (PUCPR). Docente do curso de administração da Universidade Estadual do Centro Oeste - UNICENTRO/Pr

2 Mestrado em Administração (UEL). Docente do ensino superior na área de Administração

3 Discente do curso de administração da Universidade Estadual do Centro Oeste - UNICENTRO/Pr

${ }^{4}$ Discente do curso de administração da Universidade Estadual do Centro Oeste - UNICENTRO/Pr

5 Discente do curso de administração da Universidade Estadual do Centro Oeste - UNICENTRO/Pr

RECIMA21 - Ciências Exatas e da Terra, Sociais, da Saúde, Humanas e Engenharia/Tecnologia
} 


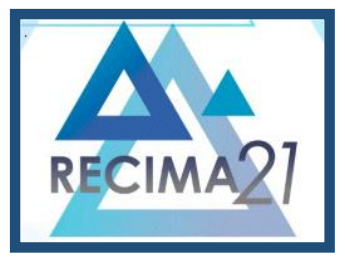

\section{RECIMA21 - REVISTA CIENTÍFICA MULTIDISCIPLINAR ISSN 2675-6218}

at home. Based on the results obtained, it was possible to verify that the two generations claim to have a certain knowledge about financial management, and likewise demonstrate the importance of saving for professional and personal growth.

\section{KEYWORDS: Generation Z. Generation Y. Financial education}

\section{RESUMEN}

El estudio se refiere al tema de la educación financiera y su enfoque son con las generaciones $Y$ y $Z$ y, está claro que existe una preocupación de diferentes generaciones sobre el futuro en el ámbito profesional, financiero y personal en relación con la conciencia de la educación financiera. El objetivo es analizar la opinión y actitud de las dos generaciones, $Y$ y Z, en relación a la importancia y conocimiento sobre la educación financiera. Se llevó a cabo un enfoque teórico caracterizando a las dos generaciones, sobre educación financiera y finezas personales. Como método de investigación, se realizaron colectas en ciudades del interior de Paraná mediante la aplicación de 102 cuestionarios, enviados a través de Google Forms, caracterizando el muestreo no probabilístico. Los principales hallazgos indican que el 66,7\% menciona que tiene conocimientos sobre gestión financiera y el 63,7\% tiene control sobre las finanzas personales, y el $89,2 \%$ afirma ser consciente de la importancia de la planificación para la estabilidad financiera. El 72,5\% está de acuerdo en que es posible ahorrar con el salario actual, el 41,2\% está de acuerdo en que puede acceder a información sobre inversiones en redes sociales y el $75,5 \%$ dice tener claro cuánto ahorrar mensualmente para lograr la meta propuesta. Por último, el 78,4\% coincide en que tiene conocimiento entre lo que son gastos esenciales, necesarios y superfluos, el 70,6\% menciona que es consciente de qué gastos son más importantes y el 77,5\% está de acuerdo en que son capaces de contribuir.

\section{PALABRAS CLAVE: Generación Z. Generación Y. Educación financeira}

\section{INTRODUÇÃO}

De acordo com Lucke (2014), pensar e se prevenir financeiramente não está limitado em ter as contas em dia, sem dívidas atrasadas e sem investimentos. O equilíbrio diante dessa situação pode mudar estrategicamente. Segundo Cunha e Laudares (2017), em uma sociedade que busca de modo intenso o consumo, o qual muitas vezes não reflete a realização de necessidades, mas por adição de bens ou por status. Numa abordagem importante é a educação dos jovens para uma consciente aquisição de processos e produtos inerentes às suas necessidades, de modo que os mesmos revelem a consciência de um consumo equilibrado e relevante para o futuro.

De certa forma, não é difícil extrair conhecimento sobre investimentos gastos e controle sobre educação financeira, até mesmo porque há meios que possibilitam e levam esses conhecimentos até os jovens por meio de tecnologias que estão ao alcance de todos.

Quando se fala dos jovens, é importante destacar as gerações que trabalham e convivem simultaneamente, tem os Baby Boomers (1945 e 1965), geração X (década de 60 e 70), geração Y (década de 80 até meados dos anos 90) e a geração Z, anos 2000 em diante (ZOMER; COSTA, 2018).

Cabe destacar que é importante entender quais situações levam essas gerações a se interessarem por investir e poupar perante um momento de crise e falta de comprometimento com o futuro financeiro. Pensando nessa questão, o problema de pesquisa é: qual das gerações analisadas obtém mais conhecimento e visão econômico-financeira e de que modo exercem suas habilidades?

Este estudo tem como objetivo analisar a opinião e atitude de duas gerações, $Y$ e Z, em relação à importância e ao conhecimento sobre a educação financeira. 


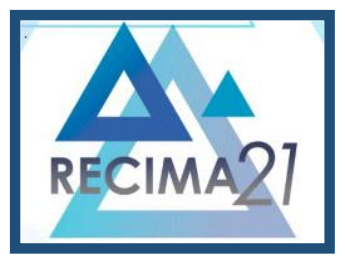

\section{RECIMA21 - REVISTA CIENTÍFICA MULTIDISCIPLINAR ISSN 2675-6218}

Como objetivos específicos tem-se como proposta levantar o quanto essas gerações: i) tem conhecimento sobre administração financeira; ii) tem controle da finanças pessoais; iii) tem conhecimento da importância de um planejamento para uma estabilidade financeira; iv) tem ciência de poupar com o salário atual; v) tem acesso às informações sobre investimentos nas redes sociais; vi) tem clareza de quanto economizar mensalmente para alcançar o objetivo; vii) tem conhecimento entre o que são gastos essenciais, necessários e supérfluos; viii) tem conhecimento de quais gastos são de maior importância; e ix) tem condições de contribuir com os gastos domiciliares em casa.

Este estudo se justifica com o intuito de apresentar qual a visão dos jovens de determinada faixa etária com relação à importância do planejamento de sua renda mensal, e como isso pode trazer vantagens futuras caso seja administrado de forma correta, e como pode afetar negativamente a pessoa, caso seja usado de forma descontrolada, podendo até trazer riscos à saúde.

Nesse sentido, o estudo tem como base investigar como os jovens vêm trabalhando e quais conhecimentos eles têm sobre a educação financeira, para investimentos do presente bem como para seus investimentos futuros. As informações colhidas são sobre faixa etária entre 18 e 35 anos, geração $Y$ e Z, tanto do gênero feminino, quanto masculino.

\section{FUNDAMENTAÇÃO TEÓRICA}

Como parte da fundamentação teórica, a abordagem trata rapidamente sobre as gerações $Y$ e $Z$, educação financeira e finanças pessoais.

\section{Geração Y e Z}

Não é novidade que o país está sempre evidenciando assuntos de momentos de crises financeiras, diante disso, o estudo feito sobre o Brasil demonstra um dos menores índices de poupança do mundo, segundo relatório do Banco Mundial. Em 2016, apenas 28\% da população declarou ter economizado alguma quantia nos últimos 12 meses (BANCO MUNDIAL, 2017).

De acordo com o Banco Mundial (2017), as condições de taxas que os bancos oferecem tornam as famílias mais vulneráveis financeiramente e sem perspectivas. As análises deste trabalho buscam contribuir com conhecimento e profundidade sobre o assunto poupar, e demonstrar o conhecimento sobre o tema, uma vez que suas conclusões apontam a relevância da orientação temporal nas tomadas de decisão. Em uma visão geral, os resultados obtidos mostram que é proveitoso fazer com que os jovens invistam em sua vida no longo prazo, pois isso thes assegura uma estabilidade e talvez até possibilite futuros investimentos e ser empreendedor sem ter que custear financiamentos que podem levar a gastos desnecessários. O conhecimento sobre um futuro financeiro pode ser atribuído por programas escolares desde o ensino mais básico, e ser adotado até uma formação mais abrangente.

Mitchell e Lusardi (2015) afirmaram que programas de educação financeira podem ser mais bemvindos entre as mulheres, uma vez que elas, além de apresentarem menor nível de conhecimento financeiro, estão mais propensas que os homens a assumir que não sabem uma resposta. Apesar dos dois públicos apresentarem pouco conhecimento sobre a administração financeira, parece que está entre 


\section{RECIMA21 - REVISTA CIENTÍFICA MULTIDISCIPLINAR ISSN 2675-6218}

COMPORTAMENTO DAS GERAÇÕES Y E Z QUANTO A IMPORTÂNCIA DA EDUCAÇÃO FINANCEIRA Cláudio Luiz Chiusoli, Thiago Ferreira Spiri, Ana Lucia Mendes, Bruna Volski dos Santos, Laryssa Latczuk Eurich

as mulheres os conhecimentos mais aprofundados, pois obtém mais expectativas para a busca de novos conhecimentos.

Assim, o consumidor mais jovem, a geração Z, evolui juntamente com a revolução tecnológica, com o passar do tempo, da mesma forma como cresceu a competição entre as marcas, tecnologias e empresas, compreender os desejos e necessidades dessa geração tornou-se essencial para as organizações (CHIUSOLI et al., 2020).

A geração $Y$ tem particularidades, frequentemente buscam o estigma de qualidade de vida acima de tudo e não são leais às empresas onde trabalham, apresentando pouco comprometimento para assumir cargos de liderança; por isso, este estudo se justifica em compreender as diferenças dessas gerações (LIMA RIBEIRO; CHIUSOLI, 2020).

\section{Educação financeira}

De acordo com Potrick et al. (2014), a alfabetização financeira ou educação financeira como é conhecida no Brasil, vem obtendo dificuldades no modo de repassar conhecimentos sobre o tema, como passar o conceito e fazer com que o objetivo de ensinar seja propício ao entendimento. Uma vez que a alfabetização financeira vai além de educação financeira, considerando que a alfabetização financeira é conceituada por meio da combinação de três variáveis, quais sejam: conhecimento financeiro, atitude financeira e comportamento financeiro.

Teixeira (2015) ressalta que a educação financeira não vem do modo de aprender a economizar, cortar gastos desnecessários ou buscar meios de se manter estável, é muito além disso. É buscar meios de se manter um modo de vida saudável financeiramente tanto no presente quanto no futuro, obtendo uma segurança material necessária e uma garantia para eventuais imprevistos futuros e mesmo assim obtendo no seu dia a dia uma vida socialmente estável sem regressões ou submeter a situações inferiores do cotidiano.

Portanto, é possível analisar que o simples conhecimento sobre finanças não quer dizer que o indivíduo seja alfabetizado financeiramente, poupar não é o mesmo que praticar, pois o indivíduo pode obter conhecimento necessário e mesmo assim não saber o que é poupar financeiramente e sucessivamente alcançar metas no que condiz com o planejamento.

E ganhar dinheiro não se restringe somente ao que é ganho pelo trabalho, mas as pessoas que têm o hábito de poupar regularmente, mesmo pequenas quantias, estão no caminho do sucesso (SARAIVA, 2017).

\section{Finanças Pessoais}

Finanças pessoais é um assunto bastante discutido ultimamente, decorrente da sua importância em qualquer decisão financeira tanto pessoal quanto familiar, considerando-se que uma decisão correta pode trazer inúmeros benefícios à pessoa ou a família, enquanto, se mal planejada, pode acarretar o contrário, comprometendo a vida social estável da família.

Muitas vezes, a falta de conhecimento desde o ensino fundamental pode comprometer o indivíduo em vários aspectos, pois desde infância os filhos já veem seus pais planejando algo e sucessivamente já 


\section{RECIMA21 - REVISTA CIENTÍFICA MULTIDISCIPLINAR ISSN 2675-6218}

COMPORTAMENTO DAS GERAÇÕES Y E Z QUANTO A IMPORTÂNCIA DA EDUCAÇÃO FINANCEIRA Cláudio Luiz Chiusoli, Thiago Ferreira Spiri, Ana Lucia Mendes, Bruna Volski dos Santos, Laryssa Latczuk Eurich

vem obtendo um pequeno conhecimento sobre finanças devido elas estarem presentes na vida do ser humano.

De acordo com Santos, Moreira e Silva (2018), finanças pessoais estão ligadas a planejamento sobre gastos, financiamentos, gerenciamento de plano de aposentadoria e investimentos futuros, visando um equilíbrio e estabilização financeira, para isso o indivíduo conta com a ajuda de uma conta corrente, ferramenta do PDCA, que nada mais é do que um planejamento estratégico que contribui para um devido acúmulo de bens e riquezas e que visa a formação do patrimônio próprio.

Esse modo estratégico foi muito utilizado em empresas, porém com a necessidade de estabelecerse financeiramente as pessoas vem aderindo, visando um modelo de planejar suas vidas e estabilizar recursos, avaliar o desempenho e maximizar seus resultados.

O planejamento financeiro é importante para uma estabilização pessoal e isso depende de pessoa para pessoa, pelo objetivo que cada um almeja tanto a curto como a longo prazo. A falta de conhecimento da população faz com que tomem decisões erradas por falta de planejar, o que deixa o indivíduo sem saber como ganhar, gastar e investir seus recursos (BRAIDO, 2014).

\section{RESULTADO E DISCUSSÃO}

Nessa seção realiza-se a análise dos resultados e discussão, sendo composta por 11 Quadros com segmentação por gênero e faixa etária (gerações $Z$ e Y). No Quadro 1 tem-se primeiramente os dados pessoais; e após, do quadro 2 ao 10, o comportamento das gerações $Y$ e $Z$ estudadas referente aos objetivos propostos. Por fim, o Quadro 11 é referente aos resultados do teste Qui-Quadrado, para mostrar a rejeição ou não das hipóteses $\mathrm{H} 0$ e $\mathrm{H} 1$ investigadas. Assim, destaca-se quanto ao gênero dos entrevistados, que a maior parte são mulheres, com $57,8 \%$, e por meio do Quadro 1 indica-se que, entre as mulheres, $71,2 \%$ são da geração $Z$.

Quadro 1 - Perfil do entrevistado: geração Z e Y
\begin{tabular}{|l|c|c|c|}
\hline Geração & Feminino & Masculino & Total \\
\hline Geração Z & $71,2 \%$ & $60,5 \%$ & $66,7 \%$ \\
\hline Geração Y & $28,8 \%$ & $39,5 \%$ & $33,3 \%$ \\
\hline Total & $100,0 \%$ & $100,0 \%$ & $100,0 \%$ \\
\hline
\end{tabular}

Fonte: dados da pesquisa (2020)

O Quadro 2 refere-se à variável "Tenho conhecimento sobre administração financeira"; o qual mostra um p-valor de 0,904 para a hipótese $\mathrm{H} 0$ e um p-valor de 0,077 para a hipótese H1. O teste do Qui Quadrado sugere que as hipóteses $\mathrm{H} 0$ e $\mathrm{H} 1$ não devem ser rejeitadas, ou seja, na segmentação por gênero e perfil quanto a geração, não apresenta diferença significativa entre as respostas.

De acordo com a abordagem do conhecimento sobre administração financeira, verifica-se que cerca de $66,7 \%$ declararam possuir, proporção levemente superior entre a geração $Y$, com $70,6 \%$, e as mulheres, com $67,8 \%$.

Considerando os achados, Mitchell e Lusardi (2015) afirmaram que programas de educação financeira podem ser mais bem-vindos entre as mulheres, uma vez que elas, além de apresentarem menor 


\section{RECIMA21 - REVISTA CIENTÍFICA MULTIDISCIPLINAR ISSN 2675-6218}

COMPORTAMENTO DAS GERAÇÕES Y E Z QUANTO A IMPORTÂNCIA DA EDUCAÇÃO FINANCEIRA Cláudio Luiz Chiusoli, Thiago Ferreira Spiri, Ana Lucia Mendes, Bruna Volski dos Santos, Laryssa Latczuk Eurich

nível de conhecimento financeiro, estão mais propensas que os homens a assumirem que não sabem uma resposta.

Quadro 2 - Tenho conhecimento sobre administração financeira
\begin{tabular}{|l|c|c|c|c|c|}
\hline Escala & Feminino & Masculino & Geração Z & Geração Y & Total \\
\hline Concordo & $67,8 \%$ & $65,1 \%$ & $64,7 \%$ & $70,6 \%$ & $66,7 \%$ \\
\hline Indiferente & $15,3 \%$ & $18,6 \%$ & $13,2 \%$ & $23,5 \%$ & $16,7 \%$ \\
\hline Discordo & $16,9 \%$ & $16,3 \%$ & $22,1 \%$ & $5,9 \%$ & $16,7 \%$ \\
\hline Total Geral & $100,0 \%$ & $100,0 \%$ & $100,0 \%$ & $100,0 \%$ & $100,0 \%$ \\
\hline
\end{tabular}

Fonte: dados da pesquisa (2020)

O Quadro 3 refere-se à variável "Tenho controle das finanças pessoais"; mostrando um p-valor de 0,919 para a hipótese $\mathrm{H0}$ e um p-valor de 0,393 para a hipótese H1. Assim, por meio do teste Qui Quadrado indica-se que as hipóteses $\mathrm{H} 0$ e $\mathrm{H} 1$ não devem ser rejeitadas.

Nesse questionamento, 63,7\% declararam que têm controle sobre as finanças pessoais, em que a proporção é maior com $65,1 \%$ no grupo masculino e $67,6 \%$ entre os entrevistados da geração $Y$. Tais resultados com maiores índices entre os grupos de participantes citados reforçam o exposto por Santos, Moreira e Silva (2018) que finanças pessoais estão ligadas a planejamento sobre gastos, financiamento, plano de aposentadoria e investimentos futuros, visando um equilíbrio e estabilização financeira.

\begin{tabular}{|l|} 
Quadro 3 - Tenho controle das finanças pessoais \\
\begin{tabular}{|l|l|l|l|l|l|}
\hline Escala & Feminino & Masculino & Geração Z & Geração Y & Total \\
\hline Concordo & $62,7 \%$ & $65,1 \%$ & $61,8 \%$ & $67,6 \%$ & $63,7 \%$ \\
\hline Indiferente & $20,3 \%$ & $20,9 \%$ & $19,1 \%$ & $23,5 \%$ & $20,6 \%$ \\
\hline Discordo & $16,9 \%$ & $14,0 \%$ & $19,1 \%$ & $8,8 \%$ & $15,7 \%$ \\
\hline Total Geral & $100,0 \%$ & $100,0 \%$ & $100,0 \%$ & $100,0 \%$ & $100,0 \%$ \\
\hline
\end{tabular}
\end{tabular}

Fonte: dados da pesquisa (2020)

O Quadro 4 refere-se à variável "Tenho conhecimento da importância do planejamento para estabilidade financeira"; mostrando um p-valor de 0,837 para a hipótese H0 e um p-valor de 0,657 para a hipótese $\mathrm{H} 1$, dessa forma, as hipóteses $\mathrm{H} 0$ e H1 não devem ser rejeitadas, ou seja, na segmentação por gênero e perfil quanto a geração, não apresenta diferença significativa entre as respostas.

Quando questionados se estão cientes que é necessário o planejamento financeiro para ter estabilidade no futuro, os números sobem para 89,2\%, com índices muitos próximos entre cruzamento por gênero e as gerações. Os resultados obtidos reforçam que o planejamento financeiro é importante para uma estabilização pessoal e isso depende de pessoa para pessoa em relação ao objetivo almejado tanto a curto ou longo prazo (BRAIDO, 2014).

Quadro 4 - Tenho conhecimento da importância do planejamento para estabilidade financeira
\begin{tabular}{|l|c|c|c|c|c|}
\hline Escala & Feminino & Masculino & Geração Z & Geração Y & Total \\
\hline Concordo & $88,1 \%$ & $90,7 \%$ & $89,7 \%$ & $88,2 \%$ & $89,2 \%$ \\
\hline Indiferente & $10,2 \%$ & $7,0 \%$ & $7,4 \%$ & $11,8 \%$ & $8,8 \%$ \\
\hline Discordo & $1,7 \%$ & $2,3 \%$ & $2,9 \%$ & $0,0 \%$ & $2,0 \%$ \\
\hline Total Geral & $100,0 \%$ & $100,0 \%$ & $100,0 \%$ & $100,0 \%$ & $100,0 \%$ \\
\hline
\end{tabular}

Fonte: dados da pesquisa (2020)

RECIMA21 - Ciências Exatas e da Terra, Sociais, da Saúde, Humanas e Engenharia/Tecnologia 


\section{RECIMA21 - REVISTA CIENTÍFICA MULTIDISCIPLINAR ISSN 2675-6218}

COMPORTAMENTO DAS GERAÇÕES Y E Z QUANTO A IMPORTÂNCIA DA EDUCAÇÃO FINANCEIRA Cláudio Luiz Chiusoli, Thiago Ferreira Spiri, Ana Lucia Mendes, Bruna Volski dos Santos, Laryssa Latczuk Eurich

O Quadro 5 refere-se à variável "É possível fazer poupança com o salário atual"; mostrando um pvalor de 0,331 para a hipótese $\mathrm{H} 0$ e um p-valor de 0,690 para a hipótese $\mathrm{H} 1$, o que indica que as hipóteses H0 e H1 não devem ser rejeitadas, ou seja, na segmentação por gênero e perfil quanto a geração, não se apresentam diferenças significativas entre as respostas.

Nessa questão, com a remuneração atual, cerca de $72,5 \%$ deram uma resposta positiva sobre ser possível poupar, e esse índice é maior entre o grupo de mulheres (78\%) e ligeiramente maior também entre os de geração Z (73,5\%). Apesar da importância de poupar, estudos realizados mostram que o Brasil possui um dos menores índices de poupança do mundo, onde apenas $28 \%$ da população declarou ter economizado alguma quantia nos últimos 12 meses (BANCO MUNDIAL, 2017).

Quadro 5 - É possível fazer poupança com o salário atual

\begin{tabular}{|l|c|c|c|c|c|}
\hline Escala & Feminino & Masculino & Geração Z & Geração Y & Total \\
\hline Concordo & $78,0 \%$ & $65,1 \%$ & $73,5 \%$ & $70,6 \%$ & $72,5 \%$ \\
\hline Indiferente & $10,2 \%$ & $18,6 \%$ & $14,7 \%$ & $11,8 \%$ & $13,7 \%$ \\
\hline Discordo & $11,9 \%$ & $16,3 \%$ & $11,8 \%$ & $17,6 \%$ & $13,7 \%$ \\
\hline Total Geral & $100,0 \%$ & $100,0 \%$ & $100,0 \%$ & $100,0 \%$ & $100,0 \%$ \\
\hline
\end{tabular}

Fonte: dados da pesquisa (2020)

O Quadro 6 refere-se à variável é "Consigo ter acesso às informações sobre investimentos nas redes sociais"; mostrando um p-valor de 0,326 para a hipótese $\mathrm{H} 0$ e um p-valor de 0,223 para a hipótese $\mathrm{H} 1$, o que indica que as hipóteses $\mathrm{H} 0$ e $\mathrm{H} 1$ não devem ser rejeitadas, ou seja, na segmentação por gênero e perfil quanto a geração, não se apresentam diferenças significativas entre as respostas.

Quando questionados se as redes sociais proporcionam informações sobre investimentos, os números sofrem uma queda para $41,2 \%$ dos entrevistados como a percepção de quem tem esse acesso. O índice é destaque entre os de geração $\mathrm{Y}$, com 52,9\% e entre as mulheres, com 45,8\% de concordância. Baseado nos indicadores, embora não tão altos, Goulart (2014) comenta que redes sociais são espaços que facilitam a interação entre pessoas, com o objetivo de compartilhar questões pessoais ou profissionais e que é possível achar informações de toda natureza, seja em blogs, comunidades de conteúdo, chats, Facebook, Instagram e outros.

Quadro 6 - Consigo ter acesso às informações sobre investimentos nas redes sociais
\begin{tabular}{|l|c|c|c|c|c|}
\hline Escala & Feminino & Masculino & Geração Z & Geração Y & Total \\
\hline Concordo & $45,8 \%$ & $34,9 \%$ & $35,3 \%$ & $52,9 \%$ & $41,2 \%$ \\
\hline Indiferente & $32,2 \%$ & $30,2 \%$ & $35,3 \%$ & $23,5 \%$ & $31,4 \%$ \\
\hline Discordo & $22,0 \%$ & $34,9 \%$ & $29,4 \%$ & $23,5 \%$ & $27,5 \%$ \\
\hline Total Geral & $100,0 \%$ & $100,0 \%$ & $100,0 \%$ & $100,0 \%$ & $100,0 \%$ \\
\hline
\end{tabular}

$$
\text { Fonte: dados da pesquisa (2020) }
$$

O Quadro 7 refere-se à variável "Tenho clareza de quanto economizar mensalmente para alcançar meu objetivo"; mostrando um p-valor de 0,036 para a hipótese $\mathrm{HO}$ e um p-valor de 0,492 para a hipótese H1. Assim, de acordo com o teste do Qui Quadrado, a hipótese H0 deve ser rejeitada e a hipótese H1 não deve ser rejeitada. 


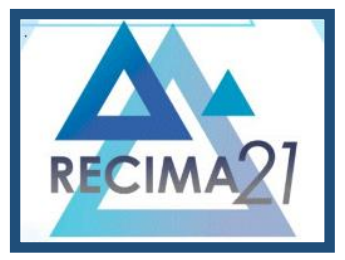

\section{RECIMA21 - REVISTA CIENTÍFICA MULTIDISCIPLINAR ISSN 2675-6218}

Ao serem questionados se havia a noção de quanto economizar mensalmente para alcançar o objetivo pessoal, no geral, $75,5 \%$ concordaram. No entanto, destaque pelas diferenças estatísticas verificadas é que, entre os homens, o índice é de $88,4 \%$ contra $66,1 \%$ das mulheres quanto à concordância. E entre as gerações, apesar de não apresentarem diferenças significativas, o destaque para essa variável é entre os de geração $\mathrm{Y}, \operatorname{com} 82,4 \%$.

Os resultados obtidos, com um alto índice de concordância, vêm de acordo com o postulado por Teixeira (2015), ao afirmar que a educação financeira não vem do modo de aprender a economizar, cortar gastos desnecessários ou buscar meios de se manter estável, devendo ir além disso. É possível que muito desse aprendizado tenha origem na própria orientação dos pais.

Quadro 7 - Tenho clareza de quanto economizar mensalmente para alcançar meu objetivo
\begin{tabular}{|l|c|c|c|c|c|}
\hline Escala & Feminino & Masculino & Geração Z & Geração Y & Total \\
\hline Concordo & $66,1 \%$ & $88,4 \%$ & $72,1 \%$ & $82,4 \%$ & $75,5 \%$ \\
\hline Indiferente & $20,3 \%$ & $7,0 \%$ & $16,2 \%$ & $11,8 \%$ & $14,7 \%$ \\
\hline Discordo & $13,6 \%$ & $4,7 \%$ & $11,8 \%$ & $5,9 \%$ & $9,8 \%$ \\
\hline Total Geral & $100,0 \%$ & $100,0 \%$ & $100,0 \%$ & $100,0 \%$ & $100,0 \%$ \\
\hline
\end{tabular}

Fonte: dados da pesquisa (2020)

O Quadro 8 refere-se à questão "Tenho conhecimento entre o que são gastos essenciais, necessários e supérfluos"; mostrando um p-valor de 0,801 para a hipótese $\mathrm{H} 0$ e um p-valor de 0,085 para a hipótese $\mathrm{H} 1$. Ambas as hipóteses $\mathrm{H} 0$ e $\mathrm{H} 1$ não devem ser rejeitadas, pelo fato que, na segmentação por gênero e geração não se apresentam diferenças significativas entre as respostas.

Como resultados, $78,4 \%$ dizem saber diferenciar os tipos de gastos e o destaque é entre os entrevistados da geração $Y(91,2 \%)$ e os homens $(81,4 \%)$. Dessa forma, destaca-se que os dados obtidos sugerem uma conscientização sobre o tema estudado, considerando que, além do ganho pela remuneração no trabalho, constaram também o hábito de poupar regularmente, mesmo em pequenas quantias, evitando assim despesas desnecessárias (SARAIVA, 2017).

Quadro 8 - Tenho conhecimento entre o que são gastos essenciais, necessários e supérfluos
\begin{tabular}{|l|l|l|l|l|l|}
\hline Escala & Feminino & Masculino & Geração Z & Geração Y & Total \\
\hline Concordo & $76,3 \%$ & $81,4 \%$ & $72,1 \%$ & $91,2 \%$ & $78,4 \%$ \\
\hline Indiferente & $13,6 \%$ & $11,6 \%$ & $16,2 \%$ & $5,9 \%$ & $12,7 \%$ \\
\hline Discordo & $10,2 \%$ & $7,0 \%$ & $11,8 \%$ & $2,9 \%$ & $8,8 \%$ \\
\hline Total Geral & $100,0 \%$ & $100,0 \%$ & $100,0 \%$ & $100,0 \%$ & $100,0 \%$ \\
\hline
\end{tabular}

O Quadro 9 refere-se à variável "Tenho conhecimento de quais gastos são de maior importância"; mostrando um p-valor de 0,323 para a hipótese $\mathrm{H} 0$ e um p-valor de 0,702 para a hipótese H1. Em ambas as hipóteses $\mathrm{H} 0$ e H1, o teste do Qui Quadrado sugere que não devem ser rejeitadas.

Nessa questão, ao serem abordados se tem conhecimento sobre quais gastos são os mais importantes, $70,6 \%$ citam que sim. O percentual é maior junto ao público masculino, com $67,8 \%$ e no grupo da geração $\mathrm{Y}$, com $73,5 \%$. 


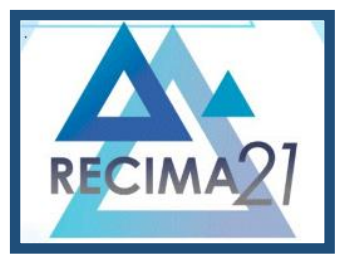

\section{RECIMA21 - REVISTA CIENTÍFICA MULTIDISCIPLINAR ISSN 2675-6218}

Tendo em vista a demonstração da importância de controlar os gastos, o assunto sobre educação financeira trata-se de um conjunto de atividades, como o controle diário das despesas, cartão de crédito, financiamentos e empréstimos, assim, essa consciência torna-se fundamental (CORDEIRO et al., 2018).

Quadro 9 - Tenho conhecimento de quais gastos são de maior importância

\begin{tabular}{|l|c|c|c|c|c|}
\hline Escala & Feminino & Masculino & Geração Z & Geração Y & Total \\
\hline Concordo & $67,8 \%$ & $74,4 \%$ & $69,1 \%$ & $73,5 \%$ & $70,6 \%$ \\
\hline Indiferente & $15,3 \%$ & $18,6 \%$ & $16,2 \%$ & $17,6 \%$ & $16,7 \%$ \\
\hline Discordo & $16,9 \%$ & $7,0 \%$ & $14,7 \%$ & $8,8 \%$ & $12,7 \%$ \\
\hline Total Geral & $100,0 \%$ & $100,0 \%$ & $100,0 \%$ & $100,0 \%$ & $100,0 \%$ \\
\hline
\end{tabular}

Fonte: dados da pesquisa (2020)

O Quadro 10 refere-se à variável "Tenho condições de contribuir com os gastos domiciliares em casa"; mostra um p-valor de 0,041 para a hipótese H0 e um p-valor de 0,115 para a hipótese H1. Conforme teste estatístico, sugere que a hipótese $\mathrm{H} 0$ deve ser rejeitada, podendo destacar resultados distintos entre homens e mulheres e a hipótese $\mathrm{H} 1$ não deve ser rejeitada, pois no perfil quanto a geração, não se apresentam diferenças significativas na proporção das respostas.

E por fim, o último assunto aborda se há condições de contribuir com gastos domiciliares, e 77,5\% dizem possuir. O grupo que cita ter maiores condições são os homens, com $86 \%$, contra a opinião de $71,2 \%$, o que justifica pelo resultado do teste Qui Quadrado que indica diferença estatística. Em relação ao perfil quanto a geração, aqueles que se enquadram na geração $\mathrm{Y}$, o índice é de $88,2 \%$ contra $72,1 \%$ da reposta da geração $Z$.

Nesse contexto, os resultados reforçam que o orçamento familiar é a base de toda estrutura financeira doméstica e é importante que toda a família esteja engajada nesse processo, pois a participação de todos é de extrema importância para sua elaboração orçamentária (LUZ et al., 2019).

Quadro 10 - Tenho condições de contribuir com os gastos domiciliar em casa

\begin{tabular}{|l|c|c|c|c|c|}
\hline Escala & Feminino & Masculino & Geração Z & Geração Y & Total \\
\hline Concordo & $71,2 \%$ & $86,0 \%$ & $72,1 \%$ & $88,2 \%$ & $77,5 \%$ \\
\hline Indiferente & $10,2 \%$ & $11,6 \%$ & $11,8 \%$ & $8,8 \%$ & $10,8 \%$ \\
\hline Discordo & $18,6 \%$ & $2,3 \%$ & $16,2 \%$ & $2,9 \%$ & $11,8 \%$ \\
\hline Total Geral & $100,0 \%$ & $100,0 \%$ & $100,0 \%$ & $100,0 \%$ & $100,0 \%$ \\
\hline
\end{tabular}

Fonte: dados da pesquisa (2020)

Em resumo, destacam-se por meio da Quadro 11 os resultados quanto ao teste Qui Quadrado, no qual são apontados se devem ou não serem rejeitadas ao nível de significância de $1 \%(p \leq 0,01)$ e $5 \%$ ( $p$ $\leq 0,05$ ), ao considerar as hipóteses $\mathrm{H} 0$ e $\mathrm{H} 1$. 


\section{RECIMA21 - REVISTA CIENTÍFICA MULTIDISCIPLINAR ISSN 2675-6218}

COMPORTAMENTO DAS GERAÇÕES Y E Z QUANTO A IMPORTÂNCIA DA EDUCAÇÃO FINANCEIRA Cláudio Luiz Chiusoli, Thiago Ferreira Spiri, Ana Lucia Mendes, Bruna Volski dos Santos, Laryssa Latczuk Eurich

Quadro 11 - Resumo do teste estatístico não paramétrico: Qui Quadrado
\begin{tabular}{|l|c|c|c|c|}
\hline Variáveis & P-valor & Teste hipótese H0 Gênero & P-valor & Teste hipótese H1 Geração \\
\hline Quadro 2 & 0,904 & Não rejeitar H0 & 0,077 & Não rejeitar H1 \\
\hline Quadro 3 & 0,919 & Não rejeitar H0 & 0,393 & Não rejeitar H1 \\
\hline Quadro 4 & 0,837 & Não rejeitar H0 & 0,657 & Não rejeitar H1 \\
\hline Quadro 5 & 0,331 & Não rejeitar H0 & 0,690 & Não rejeitar H1 \\
\hline Quadro 6 & 0,326 & Não rejeitar H0 & 0,223 & Não rejeitar H1 \\
\hline Quadro 7 & $0,036^{* *}$ & Rejeitar H0 & 0,492 & Não rejeitar H1 \\
\hline Quadro 8 & 0,801 & Não rejeitar H0 & 0,085 & Não rejeitar H1 \\
\hline Quadro 9 & 0,323 & Não rejeitar H0 & 0,702 & Não rejeitar H1 \\
\hline Quadro 10 & $0,041^{* *}$ & Rejeitar H0 & 0,115 & Não rejeitar H1 \\
\hline
\end{tabular}

Fonte: autores (2020) - significativo a $1 \%(p \leq 0,01)^{\star}$ e $5 \%(p \leq 0,05)^{\star \star}$

\section{CONSIDERAÇÕES FINAIS}

O estudo entregou resultados para atingir o objetivo proposto, uma vez que analisou a opinião e atitude das duas gerações, Y e Z, em relação a importância e o conhecimento sobre a educação financeira.

Como visão geral, a alfabetização financeira adequada proporcionará aos jovens e as futuras gerações uma vida financeira estável. Assim como a forma de conduzir as finanças pessoais, pois dependendo da decisão tomada, afeta tanto positivamente quanto negativamente, não só a pessoa, mas também os membros da família.

Como principais achados, destaca-se em resumo os resultados das variáveis estudadas quanto ao grau de concordância e a aplicação do teste Qui Quadrado em relação às hipóteses, se devem ou não serem rejeitadas:

- 66,7\% concordam que têm conhecimento sobre administração financeira, logo, as hipóteses H0

e $\mathrm{H} 1$ não devem ser rejeitadas;

-63,7\% concordam que têm controle das finanças pessoais, logo, as hipóteses H0 e H1 não devem ser rejeitadas;

- $89,2 \%$ concordam que têm conhecimento que é importante um planejamento para uma estabilidade financeira, logo, as hipóteses $\mathrm{H} 0$ e $\mathrm{H} 1$ não devem ser rejeitadas;

- 72,5\% concordam que é possível fazer poupança com o salário atual, logo, as hipóteses H0 e H1 não devem ser rejeitadas;

- 41,2\% concordam que conseguem ter acesso às informações sobre investimentos nas redes sociais, logo, as hipóteses $\mathrm{H} 0$ e $\mathrm{H} 1$ não devem ser rejeitadas;

- 75,5\% concordam que têm clareza de quanto economizar mensalmente para alcançar seu objetivo, logo, a hipótese $\mathrm{H} 0$ deve ser rejeitada e a hipótese $\mathrm{H} 1$ não deve ser rejeitada;

- 78,4\% concordam que têm conhecimento entre o que são gastos essenciais, necessários e supérfluos, logo, as hipóteses $\mathrm{H} 0$ e H1 não devem ser rejeitadas;

-70,6\% concordam que têm conhecimento de quais gastos são de maior importância, logo, as hipóteses $\mathrm{H} 0$ e $\mathrm{H} 1$ não devem ser rejeitadas;

- 77,5\% concordam que têm condições de contribuir com os gastos domiciliar em casa, logo, a hipótese $\mathrm{H} 0$ deve ser rejeitada e a hipótese $\mathrm{H} 1$ não deve ser rejeitada; 


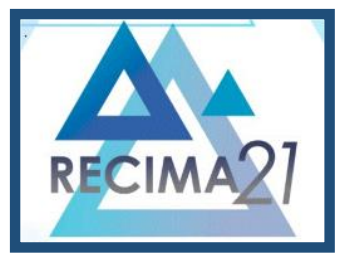

\section{RECIMA21 - REVISTA CIENTÍFICA MULTIDISCIPLINAR ISSN 2675-6218}

Como contribuição do relato de pesquisa a partir do assunto abordado no trabalho e da análise que foi feita, tem-se que a educação financeira vai muito além de apenas saber economizar, que é de extrema importância a forma com que é administrada suas finanças e que os jovens devem estar cientes da melhor forma de investimento, para evitar problemas econômicos futuramente.

Entretanto, houve algumas limitações da pesquisa, pois se trata de uma amostragem não probabilística e a análise ficou restrita ao grupo de acadêmicos pesquisados. Desse modo, sugere-se como trabalhos futuros a realização de estudos comparativos com outras gerações, bem como de outras localidades, uma vez que se trata de uma cidade do interior do Paraná.

\section{REFERÊNCIAS}

AAKER, David A. Pesquisa de Marketing. São Paulo: Atlas, 2001.

BANCO MUNDIAL. Saving for old age. Policy Research Working Paper, v. 7693, 2017.

BRAIDO, G. Planejamento financeiro pessoal dos alunos de cursos da área de gestão: estudo de uma instituição de ensino superior do rio grande do sul. Estudo \& Debate, Lajeado, v. 21, n. 1, p. 37-58, 2014.

CHIUSOLI, Cláudio Luiz et al. Atividade acadêmica, tecnologia e rede social: o comportamento da geração Z. Research, Society and Development, v. 9, n. 3, p. e169932725-e169932725, 2020.

CORDEIRO, Nilton José Neves; COSTA, Manoel Guto Vasconcelos; DA SILVA, Márcio Nascimento. Educação Financeira no Brasil: uma perspectiva panorâmica. Ensino da Matemática em Debate, v. 5, n. 1, p. $69-84,2018$.

CUNHA, Clístenes Lopes da; LAUDARES, João Bosco. Resolução de problemas na matemática financeira para tratamento de questões da educação financeira no ensino médio. Bolema: Boletim de Educação Matemática, v. 31, n. 58, p. 659-678, 2017.

FLICK, U. Introdução à metodologia de pesquisa: um guia para iniciante. São Paulo: Penso Editora, 2012.

GIL, A. C. Como elaborar projetos de pesquisa. 6. ed. São Paulo: Atlas, 2017.

GOULART, E. E. Mídias sociais: uma contribuição de análise. Porto Alegre, RS: EDIPUCRS/USCS, 2014.

KOTLER, Philip.; ARMSTRONG, Gary. Princípios de Marketing. 15. ed. São Paulo: Pearson Education do Brasil, 2015.

LIMA RIBEIRO, Atos; CHIUSOLI, Cláudio Luiz. Geração X e Y: diferenças entre o uso dos recursos tecnológicos. Revista de Administração de Empresas Eletrônica-RAEE, n. 12, p. 25-39, 2020.

LUCKE, Viviane Aparecida Caneppele et al. Comportamento financeiro pessoal: um comparativo entre jovens e adultos de uma cidade da região noroeste do estado do RS. Anais dos Seminários em Administração, v. 17, 2014.

LUZ, Elton John Ferreira; AYRES, Marcos Aurélio Cavalcante; MELO, Maria Aldiléia Silva. Orçamento Familiar: uma análise acerca da educação financeira. Humanidades \& Inovação, v. 6, n. 12, p. 206-218, 2019. 


\section{RECIMA21 - REVISTA CIENTÍFICA MULTIDISCIPLINAR ISSN 2675-6218}

COMPORTAMENTO DAS GERAÇÕES Y E Z QUANTO A IMPORTÂNCIA DA EDUCAÇ̃̃O FINANCEIRA Cláudio Luiz Chiusoli, Thiago Ferreira Spiri, Ana Lucia Mendes, Bruna Volski dos Santos, Laryssa Latczuk Eurich

MARCONI, Maria de Andrade; LAKATOS, Eva Maria. Técnicas de pesquisa: planejamento e execução de pesquisas, amostragens e técnicas de pesquisa, elaboração, análise e interpretação de dados. 8 . ed. São Paulo: Atlas, 2017.

MATTAR, F. N. Pesquisa de Marketing: metodologia, planejamento, execução e análise. 7. ed. Rio de Janeiro: Elsevier, 2014.

MITCHELL, O. S.; LUSARDI, A. Financial literacy and economic outcomes: evidence and policy implications. The Journal of Retirement, v. 3, n. 1, p. 107-114, 2015.

POTRICH A.; VIEIRA, M.; KIRCH, G. Determinantes da alfabetização financeira: proposição de um modelo e análise da influência das variáveis socioeconômicas e demográficas. Encontro nacional dos programas de pós-graduação em administração, 2014, Rio de Janeiro. Anais... Rio de Janeiro, XXXVIII ENANPAD, 2014.

SANTOS, Elaine Maria Ramos; MOREIRA, Fabiano Greter; SILVA, Luciana Codognoto. A importância do planejamento para o equilíbrio financeiro das famílias. Revista de Ciências Gerenciais, v. 22, n. 36, p. 129-133, 2018.

SARAIVA, Karla Schuck. Os sujeitos endividados e a Educação Financeira. Educar em Revista, n. 66, p. 157-173, 2017.

SIEGEL, Sidney; CASTELLAN JR, N. John. Estatística não paramétrica para as ciências do comportamento. São Paulo: Artmed-Bookman, 2017.

SILVA FILHO, Lucivaldo Lourenço da. Gestão de custos e formação de preço de venda, gestão de caixa e gestão de riscos: um estudo exploratório no arranjo produtivo local gesseiro do estado de Pernambuco. 2014. Dissertação (Mestrado) - Universidade Federal de Pernambuco, 2014.

SILVA, Dirceu; LOPES, Evandro Luiz; JUNIOR, Sérgio Silva Braga. Pesquisa quantitativa: elementos, paradigmas e definições. Revista de Gestão e Secretariado, v. 5, n. 1, p. 01-18, 2014.

TEIXEIRA, J. Um estudo diagnóstico sobre a percepção da relação entre educação financeira e matemática financeira. Tese (Doutorado em Educação Matemática) - PUCSP, São Paulo, 2015.

ZOMER, Luisa Bunn; SANTOS, Aline Regina; COSTA, Kelly Cristina de Oliveira. O perfil de alunos do curso de administração: um estudo com base nas gerações x, y e z. Revista Gestão Universitária na América Latina - GUAL, Florianópolis, p. 198-221, jun. 2018. ISSN 1983-4535. 